\title{
Diversity of Molecular Phenotypes in Acute Leukemias
}

S. Mizutani ${ }^{1}$, K. Nakamura ${ }^{1}$, M. Ozaki ${ }^{1}$, C. Tamura $^{1}$, M. Sasaki ${ }^{2}$, Y. Tsunematsu ${ }^{2}$, J. Fujimoto ${ }^{1}$, J. Hata $^{1}$, N. Kobayashi ${ }^{1}$

\section{A. Introduction}

Recent advances in immunological studies of human leukemias have enabled us to address the lineage of leukemic cells. More recent molecular studies of leukemic cells have made it possible to study the regulation of genes encoding antigen receptors, indicating there is differentiation-linked rearrangement of the receptor genes with lymphoid leukemias. We have sought to evaluate the usefulness of molecular analyses of acute leukemias on the basis of independent morphological, cytochemical, and immunological studies. These studies have led us to conclude immunophenotypically classified acute leukemias carry divergent molecular phenotypes.

\section{B. Materials and Methods}

Thirty-nine acute leukemias including 16 B- and pre-B-cell leukemias (B group), 16 $\mathrm{T}$-cell leukemias ( $\mathrm{T}$ group), and 7 mixed lineage leukemias (MLLs) were studied. Leukemic cells were obtained from heparinized peripheral blood or bone marrow. Immunophenotyping was carried out as described previously [1] by flow

This work was supported by Grant-in-Aid for General Science Research from The Ministry of Education, Science and Culture to S.M. and N.K., and for Cancer Research from The Ministry of Health and Welfare to S.M. and J.H. in Japan

1 The National Children's Medical Research Center and The National Children's Hospital, 3-35-31 Taishido, Setagaya-ku, Tokyo, Japan

${ }^{2}$ Urawa City Hospital, 2460 Mimuro, Urawa, Saitama, Japan cytometry using monoclonal antibodies CD10, CD19, CD15, CDw13, CD33, CD14, CD11 b, CD7, CD3, and CD2. Cytochemical and ultrastructural studies were also carried out according to standard methods. DNA was extracted from leukemic cells as has been described before [2]. Southern blot analysis of DNA was carried out after the cleavage of the DNAs with various restriction enzymes. DNA probes for this study include immunoglobulin heavy chain $\mathrm{JH}(\mathrm{IgJH}$, BamHI/HindIII fragment), Ig $\mu$-region genes (HM2), T-cell receptor (TCR) $\beta$ (BglII/StuI fragment of TCR $\beta$-constant region), and $\gamma(\mathrm{pH} 60)$ region genes.

\section{Results}

Thirteen out of 16 B4 and/or CALLApositive acute lymphocytic leukemias (stage II, III pre-B ALL, [3]) showed rearrangement of IgJH with or without those of TCR $\beta$ and $\gamma$ genes, which accord with the results obtained by others and ourselves previously. As 3 out of 16 B-group acute lymphoblastic leukemias (ALLs) showed no IgJH gene rearrangement, they were reprobed with $\operatorname{Ig} \mu$-chain gene. When reprobed with $\mathrm{C} \mu$-region gene, one of these three with germ line IgJH showed two Ig $\mu$-rearranged bands in addition to one germ line band, indicating this leukemia has a deletion of the $\mathrm{IgJH}$ region on both alleles. The other two cases of these three retained germ line configuration of the $\operatorname{Ig} \mu$ region as well as the IgJH region (Fig. 1, Table 1). Densitometric analysis of these germ line bands has ruled out the possibility of the deletion of $\mathrm{JH}$ and $\mathrm{C} \mu$ region of Ig heavy 
Table 1. Genomic diversity of precursor B ALLs

\begin{tabular}{lllllll}
\hline Case & Phenotype & Molecular type & \multicolumn{3}{l}{ Receptor genes } \\
\cline { 4 - 7 } & & & Ig JH & $\operatorname{Ig} \mu$ & TCR $\beta$ & $\gamma$ \\
\hline 1,2 & Stage II/III & B-stem type & G/G & G/G & G/G & G/G \\
& Stage III & Abortive pre B & D/D & R/R & G/G & G/G \\
\hline
\end{tabular}

Differentiation stage of precursor B ALLs was determined according to ref. [3]

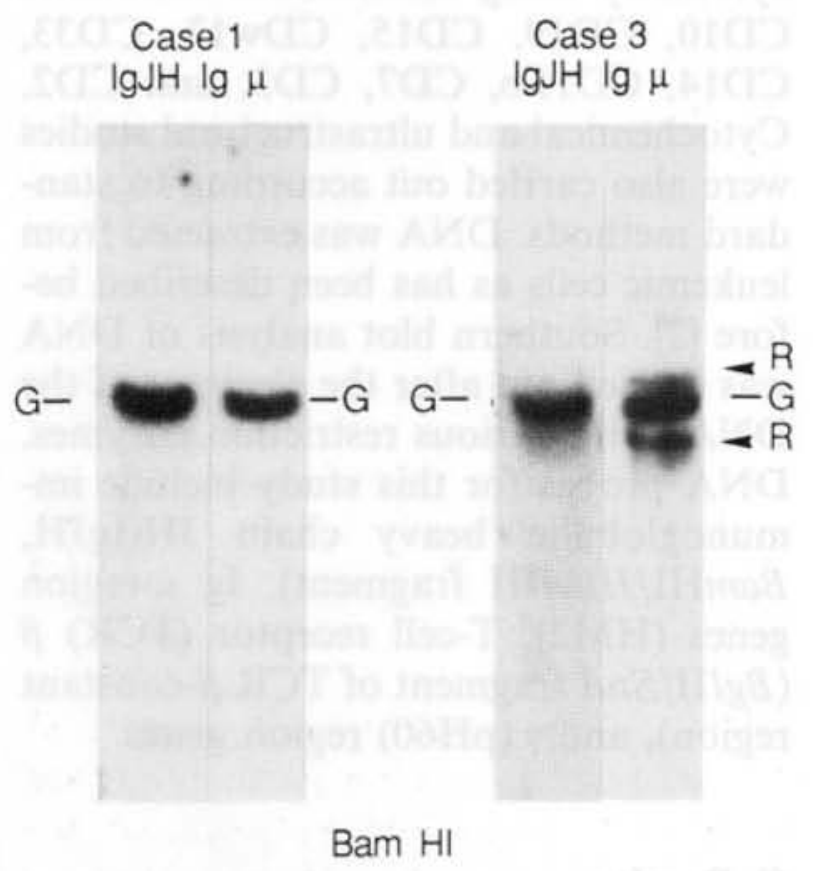

Fig. 1. $\operatorname{Ig} \mathrm{J}$ and $\mu$ gene rearrangement in case 1 and 3. DNAs from case 1 and 3 were digested with Bam HI. After electrophoresis and Southern transfer to nitrocellulose paper the blot were hybridized successively with $J$ and $\mu$ probes. The probes used are indicated above each panels. The unrearranged band is indicated by $\mathrm{G}$ and is $17 \mathrm{~kb}$ in this digest. Rearranged band is indicated by $R$.

chain locus in these leukemias (data not shown). Karyotypic analysis of one of them showed $t(4 ; 11)(q 21 ; q 23)$ acute leukemia, with which abnormality has been reported to carry a characteristic of progenitors common to B and monomacrophage. Careful examination of the cytochemical and ultrastructural features of this case showed granular positivity of $\beta$-glucuronidase, positive staining for TdT, and no ultrastructural peroxidase positivity, indicating lymphoid nature of this leukemia. Five cases among seven
MLLs were identified as having B and myeloid phenotype. Molecular analyses with antigen receptor gene probes revealed two with germ line $\mathrm{IgH}$ and TCR $\beta, \gamma$ genes, and three with rearrangement of the $\operatorname{IgH}$ gene. When referred to the morphological and cytochemical findings, these two with germ line antigen receptor genes showed Auer rods as well as strong peroxidase positivity by light microscopy (Tables 2,3 ).

\section{Discussion}

We have investigated immunological and molecular features of 39 acute leukemias; 16 B-lineage leukemias, 16 T-lineage leukemias, and 7 MLLs were identified. As the results of this study we found one pre-B type ALL with deletion of the IgJH region on both alleles, suggesting a clonal expansion of abortive pre-B-lymphocytes. The immunophenotype of this leukemia, which is consistent with that of stage III pre-B cells, suggests deletion of the $\mathrm{JH}$ region might have taken place during the rearrangement of the $\mathrm{IgH}$ region gene. The other two cases with germ line Ig JH as well as the Ig $\mu$ gene have made it possible to identify the earliest B precursor cells. The immunophenotype of these leukemias was consistent with that of stage II/III pre-B-cell ALL. In the light of this study we suggest stage II/III pre-B lymphocytes may include those with germ line IgH genes and hence probably B stem cells. Thus the careful analyses of the IgH gene of pre-B ALLs may shed light on the diversity of molecular phenotypes in B precursor ALLs. 
Table 2. Immunophenotypes of the cases presented in this study

\begin{tabular}{|c|c|c|c|c|c|c|c|c|}
\hline \multirow[b]{2}{*}{ Case } & \multicolumn{3}{|c|}{ Pre B } & \multicolumn{5}{|c|}{ MLLs with B \& M } \\
\hline & 1 & 2 & 3 & 1 & 2 & 3 & 4 & 5 \\
\hline FAB & $\mathrm{L} 2$ & L1 & $\mathrm{L} 2$ & M2 & M2 & $\mathrm{L} 2$ & $\mathrm{~L} 2$ & L2 \\
\hline HLA DR & 90 & + & 47 & 62 & 83 & + & 94 & 98 \\
\hline CD10 (CALLA) & - & 62 & 46 & - & - & 87 & - & 17 \\
\hline CD19 (B4) & 90 & + & 53 & 76 & 51 & 100 & 57 & 60 \\
\hline CD15 (LeuM1) & - & - & - & NT & 15 & NT & 14 & 37 \\
\hline CDw13 (My7) & - & - & - & 59 & 45 & - & 15 & 35 \\
\hline CD33 (My9) & - & - & - & - & 16 & NT & 23 & - \\
\hline CD14 (My4) & NT & - & NT & NT & NT & 34 & NT & NT \\
\hline CD11b (OKM1) & - & - & - & NT & - & 37 & - & NT \\
\hline CD7 (Leu9) & - & - & 19 & - & 20 & NT & - & - \\
\hline CD3 (Leu4) & - & - & NT & - & - & NT & - & - \\
\hline CD2 (Leu2) & - & NT & 15 & 23 & - & - & - & - \\
\hline SmIg & - & - & - & - & - & - & - & - \\
\hline $\mathrm{Tdt}$ & + & + & + & NT & NT & 70 & NT & NT \\
\hline
\end{tabular}

NT, not tested; - , less than $10 \%$ positivity

Only the phenotype data of precursor B ALLs with germ line IgH and of MLLs with B and myeloid phenotype are presented. The data of the other pre-B ALLs, T ALLs, and MLLs with $T$ and myeloid phenotype are omitted. Case No. 4 with $B$ and myeloid phenotype was included in this study because of the transient appearance of light microscopic positivity or peroxidase.

Table 3. Molecular phenotypes in mixed lineage leukemias with $B$ and myeloid phenotype

\begin{tabular}{llllll}
\hline Case & 1 & 2 & 3 & 4 & 5 \\
\hline IgJH & G/G & G/G & D/R & R/D & D/R \\
TCR $\gamma$ & G/G & G/G & G/G & G/G & R/R \\
TCR $\beta$ & G/G & G/G & G/G & G/G & G/G \\
\hline
\end{tabular}

Among five MLLs with B and myeloid phenotype, two cases showed no rearrangement of IgH or TCR genes. These two, with germ line antigen receptor genes, were positive for peroxidase by light microscopy, which is consistent with myeloid leukemias. Observation of Auer rods on these two leukemias made us confirm that they are bona fide myeloid leukemias. With the help of these findings, we speculate B4 antigen is not exclusively expressed on $B$ precursor cells, but can be expressed on some myeloid leukemias. As to the three cases with rearrangement of the IgH gene with or without those of TCR genes, it is not yet clear whether they are lymphoid or myeloid. But the rearrangement of anti- gen receptor genes may reflect elevated recombinase activity, suggesting they are likely to be committed to lymphoid lineage rather than to myeloid lineage. Further study is necessary to determine the nature of these leukemias.

Acknowledgments. We thank Dr. Y. Kaneko for the karyotype study of the samples, Drs. J. Shimabukuro and Y. Hayashi for referring the patient's cells to us, and Dr. Enomoto for ultramicroscopic study of the samples. We are grateful to Drs. T. Mak, P. Leder, H. Molgaard, and T. Rabbitts for molecular probes TCR $\beta, \operatorname{IgJH}, \operatorname{Ig} \mu$, and TCR $\gamma$ respectively.

\section{References}

1. Chan LC, Pegram SM, Greaves MF (1985) Contribution of immunophenotype to the classification and differential diagnosis of acute leukemia. Lancet i: 475-479

2. Ford AM, Molgaard HV, Greaves MF, Could HJ (1983) Immunoglobulin gene organisation and expression in hematopoietic stem cell leukemia. EMBO J 2:997-1001

3. Nadler LM, Korsmeyer SJ, Anderson KC et al. (1984) B cell origin of non-T cell acute lymphoblastic leukemia. J Clin Invest 74: $332-340$ 